\title{
Unraveling the complexities of invasive multimodality neuromonitoring
}

\author{
Saurabh Sinha, MD, ${ }^{1}$ Eric Hudgins, MD, PhD, ${ }^{1}$ James Schuster, MD, PhD, ${ }^{1}$ and \\ Ramani Balu, MD, PhD²
}

1'Department of Neurosurgery, Perelman School of Medicine; and 'Department of Neurology, Division of Neurocritical Care, Perelman School of Medicine, University of Pennsylvania, Philadelphia, Pennsylvania

\begin{abstract}
Acute brain injuries are a major cause of death and disability worldwide. Survivors of life-threatening brain injury often face a lifetime of dependent care, and novel approaches that improve outcome are sorely needed. A delayed cascade of brain damage, termed secondary injury, occurs hours to days and even weeks after the initial insult. This delayed phase of injury provides a crucial window for therapeutic interventions that could limit brain damage and improve outcome.

A major barrier in the ability to prevent and treat secondary injury is that physicians are often unable to target therapies to patients' unique cerebral physiological disruptions. Invasive neuromonitoring with multiple complementary physiological monitors can provide useful information to enable this tailored, precision approach to care. However, integrating the multiple streams of time-varying data is challenging and often not possible during routine bedside assessment.
\end{abstract}

The authors review and discuss the principles and evidence underlying several widely used invasive neuromonitors. They also provide a framework for integrating data for clinical decision making and discuss future developments in informatics that may allow new treatment paradigms to be developed.

https://thejns.org/doi/abs/10.3171/2017.8.FOCUS17449

KEY WORDS intracranial pressure; brain oxygen; cerebral blood flow; traumatic brain injury; multimodality monitoring; informatics; precision medicine

$\mathrm{H}$ EAD trauma, intracranial bleeding, ischemic stroke, and global cerebral hypoxia cause instantaneous brain damage but also unleash destructive waves of secondary injury that unfold over days, weeks, and even months. Little can be done to reverse the initial tissue destruction caused by these acute insults to the brain. In contrast, accurate identification of the processes that produce delayed, secondary damage enables the delivery of therapies that can limit tissue injury and markedly improve outcome. Identifying and preventing secondary brain injury is thus a guiding principle of neurocritical care.

Numerous pathophysiological processes in the brainincluding tissue swelling, dysregulated cerebral metabolism, seizures, spreading depolarizations, abnormal vascular reactivity, and neuroinflammation-cause delayed cerebral tissue injury and cell death in the days and weeks following acute brain insults. ${ }^{35,36,48}$ In addition, systemic physiological perturbations can magnify secondary brain injury and contribute to poor outcomes. These processes, occurring both in the brain and the body, leave overlap- ping but unique traces of their existence on cerebral and systemic function. Finding these traces represents a fundamental challenge in the care of critically ill brain-injured patients, in large part because both the acute insult and sedative medications required for patient management can significantly depress the level of consciousness and reduce the sensitivity of clinical neurological assessments. Without methods that allow for unambiguous classification of pathophysiological states, physicians are forced to adopt "one size fits all" guidelines that advocate maintaining uniform targets for systemic variables such as blood pressure (BP) and oxygenation and fail to account for individual differences in physiology.

Direct observation of brain function with invasive physiological monitors placed inside brain tissue or cerebral vessels can identify processes that mediate delayed injury, predict therapeutic responses, and personalize care. Invasive monitors enable continuous recordings of intracranial pressure (ICP), brain oxygenation, cerebral blood flow (CBF), and neuronal electrical activity - and additionally

ABBREVIATIONS $\mathrm{AJVDO}_{2}=$ arterial to jugular venous difference in oxygen content; $\mathrm{BP}=$ blood pressure; $\mathrm{CBF}=\mathrm{cerebral}$ blood flow; $\mathrm{CMRO} 2=\mathrm{cerebral}$ metabolic rate of oxygen; $\mathrm{CPP}=$ cerebral perfusion pressure; $\mathrm{EEG}=$ electroencephalography; ICP = intracranial pressure; $\mathrm{PbtO}_{2}=$ partial pressure of brain tissue oxygen; $\mathrm{PRx}=$ pressure reactivity index; $\mathrm{SAH}=$ subarachnoid hemorrhage; $\mathrm{SjvO}_{2}=$ jugular venous oxygen saturation; $\mathrm{TBI}=$ traumatic brain injury; TDF = thermal diffusion flowmetry. SUBMITTED July 1, 2017. ACCEPTED August 9, 2017. INCLUDE WHEN CITING DOI: 10.3171/2017.8.FOCUS17449. 
allow the repeated, direct sampling of brain interstitial fluid for biochemical measurements. The bundling of multiple monitors together can increase their predictive power, because complementary changes between modalities can be identified. In addition, because distinct secondary injury processes may have unique multimodality monitoring "signatures" (e.g., brain swelling increases ICP and lowers $\mathrm{CBF}$, whereas agitation may increase ICP and CBF concomitantly), individualized therapies targeted to specific clinical situations can be instituted. Invasive multimodality monitoring lifts the veil surrounding the "black box" of the injured brain.

This review provides a framework for understanding how invasive multimodality monitoring can impact the care of patients with acute brain injury. We will outline the physiological principles and evidence supporting many of the most widely used techniques for invasive monitoring in neurocritical care, and we will discuss methods for multivariable data visualization, organization, and analysis.

\section{Matching Metabolic Needs to Supply: A General Framework for Invasive Neuromonitoring}

Over the course of the 20th century, 3 overlapping threads facilitated the development and validation of intracranial monitoring as a means to guide care in patients with acute brain injury. First, the ability to continuously record intracranial ventricular fluid pressure ${ }^{19,34}$ established the feasibility of real-time bedside monitoring of intracranial physiology. Second, indicator dilution techniques enabled accurate measurements of cerebral perfusion in humans and demonstrated that elevated ICP leads to reduced CBF. ${ }^{28}$ Finally, studies finding that markedly elevated ICP associates with poor outcome, ${ }^{40}$ coupled with postmortem studies of patients with traumatic brain injury (TBI) showing extensive ischemic lesions, ${ }^{17,18}$ showed that prolonged intracranial hypertension can reduce cerebral perfusion and cause global ischemia. Taken together, these findings suggested that aggressive ICP control could limit secondary brain injury by improving cerebral perfusion.

Subsequent work showed that abnormalities in CBF and oxidative metabolism are far more complex. After TBI, the initial period of hypoperfusion and increased oxygen demand is followed by a drop in oxygen metabolism (cerebral metabolic rate of oxygen $\left.\left[\mathrm{CMRO}_{2}\right]\right)^{2,5,16,64}$ Unable to use oxygen, the injured brain is forced to compensate by increasing anaerobic glycolysis. Further metabolic stresses create a mismatch between delivery and consumption, ultimately leading to cell death from energy failure. Whether these specific alterations in metabolic supply and demand exist in nontraumatic acute brain injuries is unclear. Nevertheless, viewing mismatches between cerebral oxygen and glucose delivery and metabolic demand as a dominant driver of secondary brain injury remains a useful framework (Table 1). Pathological processes that increase cerebral metabolic demand may benefit from augmentation of cerebral perfusion pressure (CPP), whereas instances in which supply exceeds demand may require $\mathrm{CPP}$ reductions to minimize hemorrhagic complications.
TABLE 1. Matching of cerebral metabolic demand to supply

\begin{tabular}{lcl}
\hline \multicolumn{1}{c}{ Demand $>$ Supply } & Demand $=$ Supply & \multicolumn{1}{c}{ Demand $<$ Supply } \\
\hline Ischemia & Normal & $\begin{array}{c}\text { Functional hyperemia } \\
\text { (normal) }\end{array}$ \\
\hline Seizure & Sedation & Malignant hypertension \\
\hline $\begin{array}{l}\text { Spreading depolarization } \\
\begin{array}{l}\text { Cerebral edema, vaso- } \\
\text { spasm }\end{array}\end{array}$ & Anesthesia & Luxury perfusion \\
\hline
\end{tabular}

\section{Specific Monitoring Modalities Cerebral Oxygenation \\ Jugular Venous Oximetry}

In jugular venous oximetry, the oxygen content of blood is sampled from the cerebral venous circulation to determine the global balance between cerebral oxygen delivery and metabolism. A central venous catheter is inserted into the dominant internal jugular vein and directed toward the skull until it terminates at the jugular bulb. Jugular bulb oxygen content is determined solely by cerebral oxygen consumption, because venous blood at this level has not yet mixed with blood draining from the scalp and face. Correct catheter position is determined by obtaining a lateral skull film and identifying that the tip of the catheter terminates just above the inferior border of the C-1 vertebral body. ${ }^{37,51}$

The relationship between cerebral oxidative metabolism (i.e., $\mathrm{CMRO}_{2}$ ) and $\mathrm{CBF}$ can be calculated using the Fick equation: $\mathrm{CMRO}_{2}=\mathrm{CBF}\left(\right.$ Arterial $\mathrm{O}_{2}$ content - Jugular bulb venous $\mathrm{O}_{2}$ content). Rearranging this equation shows that the ratio of $\mathrm{CMRO}_{2}$ to $\mathrm{CBF}$ equals the arterial to jugular venous difference in oxygen content $\left(\mathrm{AJVDO}_{2}\right)$. Elevated $\mathrm{AJVDO}_{2}$ values signal cerebral metabolic demand in excess of supply, whereas low $\mathrm{AJVDO}_{2}$ values suggest luxuriant perfusion or dead brain tissue. ${ }^{37,51}$

In practice, the jugular venous oxygen saturation $\left(\mathrm{SjvO}_{2}\right)$ is often used as a proxy for $\mathrm{AJVDO}_{2}$. An $\mathrm{SjvO}_{2}$ value $<55 \%$ suggests high cerebral oxygen demand or hypoperfusion, and a value $<45 \%$ is associated with increased cerebral glutamate and lactate production..$^{8,46}$ Conversely, a value $>75 \%$ suggests hyperemia or shunting around metabolically inactive dead brain tissue. Prior studies have shown robust associations between episodes of jugular venous desaturation $\left(\mathrm{SjvO}_{2}<50 \%\right)$ and poor outcomes in patients with TBI. ${ }^{8,46}$ Current guidelines from the Brain Trauma Foundation state that jugular venous oximetry monitoring can be considered to guide care and that treatment strategies that maintain $\mathrm{SjvO}_{2}$ values $>50 \%$ may improve 3 - and 6-month outcomes (Level III recommendation). ${ }^{6}$ In contrast to TBI, patients with poor outcome after diffuse hypoxic ischemic brain injury in general have elevated $\mathrm{SjvO}_{2}$ levels. ${ }^{59}$ This may be due to early cortical cell death during reperfusion, which leads to reduced overall oxygen consumption.

The $\mathrm{SjvO}_{2}$ measurements are highly sensitive to catheter position. In addition, whereas $\mathrm{SjvO}_{2}$ measurements accurately reflect the global balance between oxygen delivery and metabolic demand, they may easily miss smaller regional changes from focal injury. ${ }^{37,51}$ 


\section{Brain Tissue Oxygen Tension}

The partial pressure of brain tissue oxygen $\left(\mathrm{PbtO}_{2}\right)$ can be measured using catheters directly placed into parenchymal tissue. The most commonly used measurement system (Licox, Integra) uses a Clark electrode, which consists of an oxygen-permeable membrane that surrounds a metallic cathode and anode immersed in electrolyte solution. ${ }^{12} \mathrm{Oxy}-$ gen diffuses through this membrane and undergoes a redox reaction, resulting in an increase in current. Oxygen-induced currents rapidly reach steady state, and as a result, changes in current directly reflect fluctuations in oxygen tension.

Unlike jugular venous oximetry, $\mathrm{PbtO}_{2}$ measurements do not directly report the balance between oxygen metabolism and supply. Rather, $\mathrm{PbtO}_{2}$ reflects the product of $\mathrm{CBF}$ and arterial to venous free oxygen tension difference and provides an aggregate measure of both cerebral perfusion and systemic oxygenation. ${ }^{49}$ Despite its complicated dependence on multiple physiological factors, brain hypoxia is an independent predictor of poor outcome in brain-injured patients, ${ }^{38,44,45}$ and $\mathrm{PbtO}_{2}$ threshold-based treatment strategies may improve outcomes., ${ }^{4,41}$

Monitoring devices for $\mathrm{PbtO}_{2}$ measure local tissue oxygen tension and may not reflect global brain oxygenation. In addition, probe placement can cause local tissue injury, leading to falsely low measurements. Probe function can be assessed by measuring the response to a $100 \%$ fraction of inspired oxygen challenge.

\section{Cerebral Blood Flow}

Traditional methods for determining CBF provide single snapshot measurements and are therefore unsuitable for use as continuous monitors. In contrast, thermal diffusion flowmetry (TDF) allows for continuous real-time measurement of absolute CBF. Commercially available TDF systems (QFlow probe, Bowman Perfusion Monitor, Hemedex) measure thermal conductivity of brain tissue to quantify CBF. A catheter with 2 thermistors is inserted into parenchymal tissue. The proximal active thermistor heats adjacent tissue, while the distal passive thermistor measures parenchymal temperature. The power required to maintain a temperature difference is directly proportional to perfusion and is used to calculate blood flow. ${ }^{63}$ Cerebral blood flow measured by TDF correlates reasonably well with $\mathrm{PbtO}_{2}$ measurements in brain-injured patients, ${ }^{49,50}$ and decreases in CBF can be seen in patients with high-grade subarachnoid hemorrhage (SAH) prior to the development of delayed cerebral ischemia. ${ }^{62}$

Because thermal conductivity reflects tissue water content, TDF may also allow real-time measurements of brain tissue edema. A recent study showed a strong association between thermal conductivity and radiographic measurements of brain swelling. ${ }^{30}$ Thus, continuous determination of brain water content may provide additional information that can help guide care.

Although promising, invasive CBF measurements may require further validation before becoming widely adopted. For example, TDF monitors require repeated calibration (usually once every 30 minutes for 2-5 minutes), which leads to significant data loss. In addition, significant measurement drift can occur between calibration cycles. ${ }^{73}$ Finally, because cerebral perfusion fluctuates in response to cerebral metabolic needs, reduced CBF could simply reflect decreased metabolic demand rather than true ischemia.

\section{Cerebral Biochemistry}

Cerebral microdialysis measures biochemical markers of secondary brain damage. ${ }^{26,61} \mathrm{~A}$ double-lumen catheter surrounded by a semipermeable membrane is inserted into parenchymal tissue. Isotonic artificial CSF is continuously cycled into the inlet lumen and removed from the outlet lumen at a constant rate. Molecules in brain tissue interstitial fluid (such as metabolic byproducts, neurotransmitters, small peptides, and fatty acids) will diffuse across the semipermeable membrane, and the concentration of these analytes in the recovered dialysate can be determined. Commercially available microdialysis systems measure markers of cerebral metabolism (glucose, lactate, and pyruvate); excitotoxicity (glutamate); and cell membrane turnover (glycerol). Calculating the ratio of recovered analytes (such as lactate:pyruvate) normalizes for differences in analyte recovery and facilitates serial comparisons over time. ${ }^{26,61}$ Hourly sampling is typically used for clinical care; however, rapid sampling ${ }^{3}$ and microfluidic methods ${ }^{47}$ have been developed that enable essentially continuous measurements.

An elevated lactate/pyruvate ratio $(>40)$ is a marker of anaerobic cerebral metabolism, and reduced cerebral glucose $(<0.7 \mathrm{mmol} / \mathrm{L})$ similarly suggests a profound mismatch between metabolic demand and supply. These biochemical signatures of metabolic distress both predict poor outcome. They can occur both in response to decreases in cerebral perfusion ${ }^{9,24,26,52,54,60,61}$ and when CBF is normal. ${ }^{56,64,70 ~ " N o n i s c h e m i c " ~ m e t a b o l i c ~ c r i s e s ~ a r e ~ p r o b a b l y ~}$ due to injury-associated mitochondrial dysfunction, which reduces the brain's ability to use oxygen and triggers compensatory hyperglycolysis. ${ }^{2,16,64}$ Isolated lactate elevations could be due to neuroprotective mechanisms that provide alternative fuel sources for oxidative phosphorylation. Consistent with this view, a prior study showed that patients with SAH who had isolated lactate elevations experienced better than expected outcomes, whereas patients in whom elevated lactate coincided with brain hypoxia fared poorly ${ }^{43}$ Finally, extracellular levels of the excitatory neurotransmitter glutamate also correlate with markers of secondary brain injury and poor outcome., ${ }^{7,65}$ Seizures and reduced CPP are both associated with abnormally elevated glutamate after TBI. ${ }^{65}$

As with other invasive monitors, probe placement can induce local tissue injury, which can cause significant abnormalities in analyte levels that take hours to normalize. In addition, because of low sampling rates (usually 1 per hour) and variability in measurements, actionable changes in biochemical parameters can take hours to develop. The low time resolution of microdialysis is offset by the fact that biochemical changes can occur hours before changes in other monitored parameters can be identified. ${ }^{54}$

\section{Electrical Activity}

Seizures are common after acute brain injury and are consistently associated with worsened outcome ${ }^{10,11,69}$ Early studies evaluating the utility of continuous scalp electroencephalography (EEG) monitoring noted that seizures 

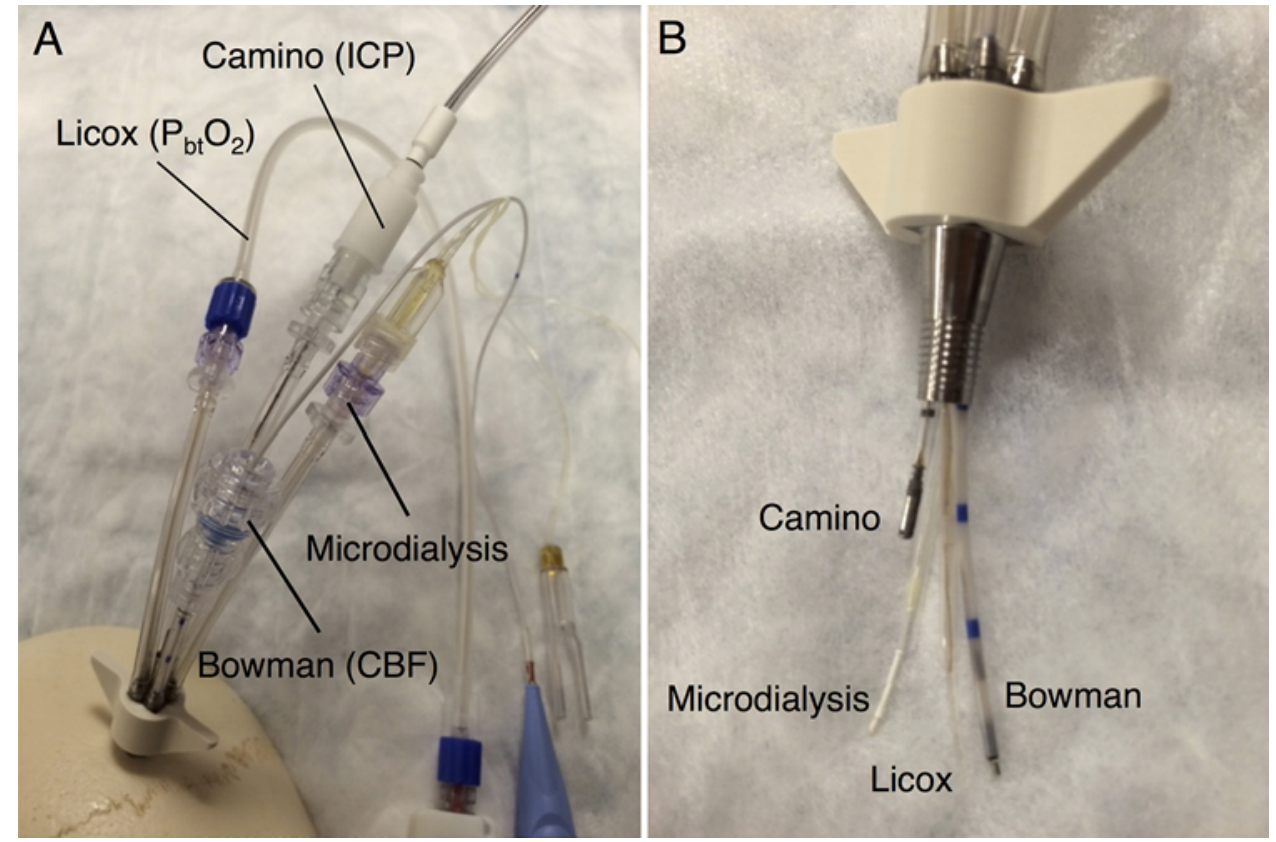

FIG. 1. Photographs showing use of a multilumen bolt housing to facilitate simultaneous placement of multiple intracranial monitors. A: External image of a quad lumen bolt showing positions of inserted Clark electrode type $\mathrm{PbtO}_{2}$ monitor (Licox, Integra); fiberoptic ICP monitor (Camino, Integra); thermal diffusion flowmetry CBF monitor (Bowman Perfusion Monitor, Hemedex); and microdialysis catheter (MDialysis). B: Image showing catheters exiting from outlets of the quad lumen bolt. The bolt lumens are oriented such that inserted catheters splay out once they are inserted into brain parenchyma.

occur in approximately $25 \%$ of patients with TBI and intracerebral hemorrhage and that the majority of seizures are nonconvulsive, with no clinical manifestations. ${ }^{10,69}$ Nonconvulsive seizures are associated with ICP increases and cerebral metabolic distress after TBI, ${ }^{68}$ independently predict hematoma expansion, ${ }^{10}$ and are associated with increased risk of hippocampal and cortical atrophy. ${ }^{25,67}$ More recent studies have used intracranial electrophysiological methods, including subdural electrocorticography and intracortical EEG, to monitor brain-injured patients. Subdural electrocorticography strips can be placed intraoperatively in patients requiring acute neurosurgical intervention, whereas depth electrodes for intracortical EEG can be inserted through a bur hole placed at bedside. Intracranial recordings detect seizures with much higher sensitivity than scalp EEG; in patients being monitored with both types of recordings, conventional scalp EEG misses up to $60 \%$ of seizures that are subsequently picked up with invasive methods. ${ }^{11,71}$ Seizures identified with invasive recordings produce brain hypoxia ${ }^{72}$ and metabolic dysregulation $^{66}$ (e.g., cerebral glycopenia and elevated lactate/pyruvate ratio), thus providing a rationale for using intracranial EEG monitoring to guide patient care.

In addition to detecting seizures with higher sensitivity, intracranial EEG allows for the detection of injuryassociated spreading depolarizations, which occur over slow time scales (minutes) and are largely undetectable by scalp EEG. ${ }^{21}$ Spreading depolarizations are waves of intense depolarization that emanate from foci of cortical injury to produce widespread metabolic distress, cytotoxic edema, and impaired neurovascular coupling. ${ }^{15,21,32}$ Spreading depolarizations are strong predictors of poor outcome after TBI, ischemic stroke, intracerebral hemorrhage, and SAH. ${ }^{15,20,21,32}$ Given that ketamine impedes the occurrence and propagation of spreading depolarizations, monitoring for these deleterious physiological stressors could positively impact patient outcome. ${ }^{23}$

\section{Integrating Multiple Modalities to Guide Patient Care}

The true promise of the techniques described in this article lies in their ability to be bundled together to provide complementary information. Streamlining monitor insertion so that multiple monitors can be placed simultaneously and proper data visualization are key prerequisites for the effective implementation of multimodality monitoring-guided care.

\section{Monitor Insertion}

The use of a multilumen bolt facilitates the simultaneous placement of multiple monitors through a single insertion site (Fig. 1). Different combinations of monitors can be placed through the same bolt housing, and in general lumens are oriented such that inserted catheters gently splay out from the insertion site. This ensures that each monitor independently samples a different brain region and reduces the risk that tissue injury around one monitor adversely affects data collected by other monitors.

\section{Data Visualization and Review}

Overlaid time series displays enable the simultaneous visualization of multiple physiological trends, which aids 

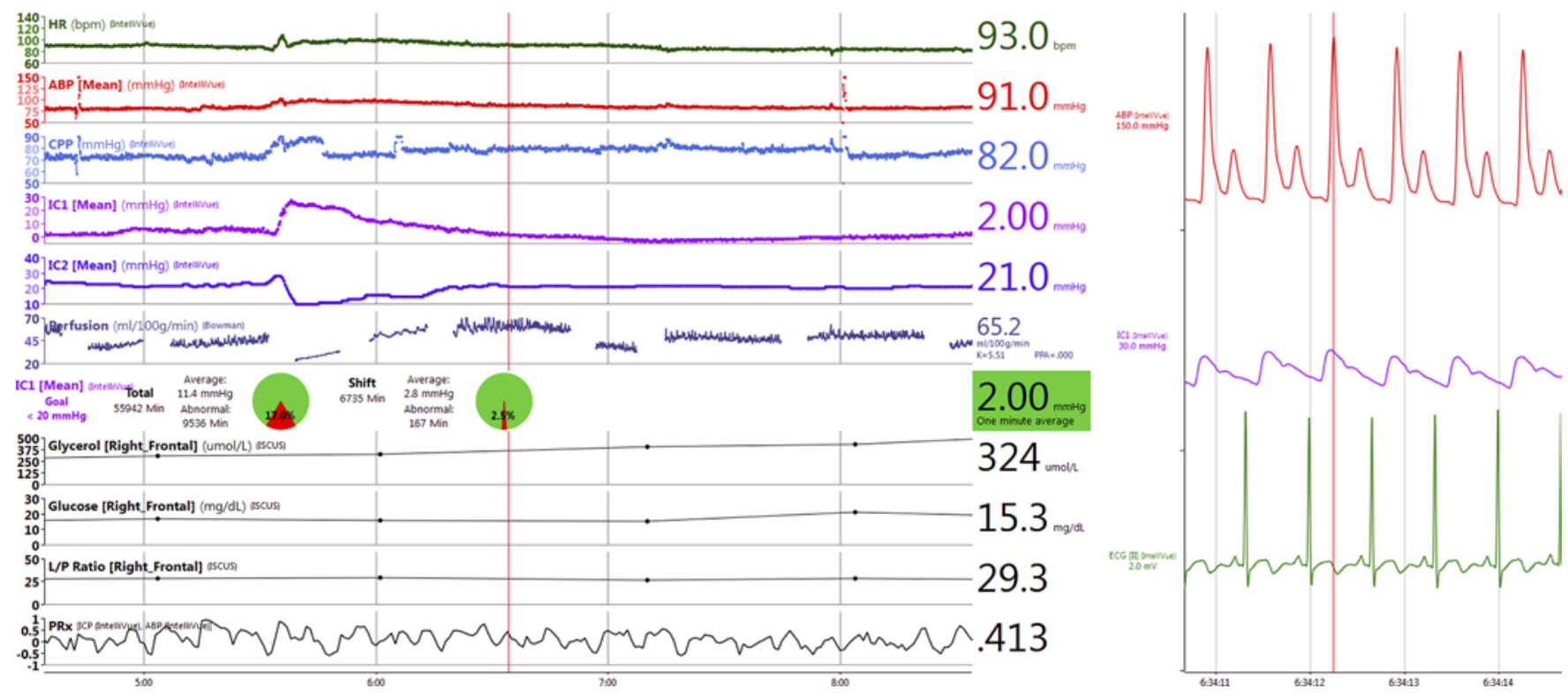

FIG. 2. Example of a multimodal monitoring display montage. This screenshot of 6 hours of data recorded from a patient with severe TBI was obtained using a commercially available multimodality analysis software package (CNS Reader, Moberg Research). In the left panel, each track shows trends in a different recorded variable. Note that IC1 refers to ICP and IC2 means $\mathrm{PbtO}_{2}$.

Between 5:00 and 6:00, there is a spontaneous rise in ICP associated with a drop in both $\mathrm{PbtO}_{2}$ and $\mathrm{CBF}$. In addition to recorded variables, one track displays ICP burden, which is defined as percentage of time that ICP is $>20 \mathrm{~mm} \mathrm{Hg}$ during monitoring (the left pie chart shows entire recording, and the right pie chart shows the current time window), while another track displays a calculated correlation index between ICP and arterial BP (the PRx). The right panel displays raw waveform data (red line marks corresponding times on the left and right panels), which aids in assessment of data fidelity. $A B P=$ arterial BP; bpm = beats per minute; $E C G=$ electrocardiogram; $H R=$ heart rate; $L / P=$ lactate/pyruvate.

in identifying concordant changes across different monitors. An example of a multimodal data display is shown in Fig. 2. Each track shows temporal trends for a specific recorded parameter over a 6-hour window. In addition to raw data, a track showing the cumulative ICP burden (the percentage of time that ICP stays $>20 \mathrm{~mm} \mathrm{Hg}$ ) is also shown. The overlay of multiple physiological trends clearly shows the effect of a spontaneous increase in ICP (denoted in the figure as IC1) on brain oxygen (denoted as IC2) and perfusion. Simultaneous display of raw waveform data (right side of the figure) allows for the identification of data artifacts (such as arterial line flushing).

Bedside trend analysis can help guide patient care; however, the large number of recorded variables makes identification of secondary injury processes difficult on bedside spot checks. Detailed retrospective review at regular intervals by a physician or technician enables identification of worrisome trends, assessment of the cumulative effectiveness of therapeutic interventions, and identification of novel patterns in the data. When conceptualized in this manner, effective analysis of invasive multimodality neuromonitoring data becomes analogous to reviewing continuous EEG data.

At our institution, we have adopted such a strategy for periodic retrospective data review (Fig. 3). Data streams from different physiological monitors are recorded and time-synchronized onto a unit (CNS Monitor, Moberg Research) that allows bedside review of physiological trends by members of the clinical team. The recorded data are also automatically archived in real time to a secure server within the hospital network that can be remotely accessed for periodic review. The retrospective identification of specific physiological signatures of secondary injury then prompts communication with the clinical team, thus closing the loop and ensuring that worrisome trends are acted on. This strategy has greatly increased the confidence among clinical staff at our institution in the utility of intracranial monitoring. Future work is required, however, to assess whether this strategy improves physiological end points or patient outcome.

\section{Moving Beyond Simple Trend Analysis: Finding Hidden Patterns}

Analyzing trends in multimodality monitoring data allows therapies to be instituted that renormalize physiology. However, our preexisting expectations for the data can severely constrain our ability to find novel physiological signatures of injury, treatment response, and outcome. For instance, because of the rich history of ICP-oriented therapies in neurocritical care, clinicians may focus on ICP elevations while ignoring other concerning patterns. Unbiased analysis methods can aid in finding hidden patterns that can guide patient care. ${ }^{22}$

\section{Pairwise Correlation Analysis}

Dependencies between physiological variables can be identified by pairwise correlation analysis. For example, plotting paired values of ICP and $\mathrm{PbtO}_{2}$ may reveal a relationship in which higher ICP is associated with lower $\mathrm{PbtO}_{2}$ 


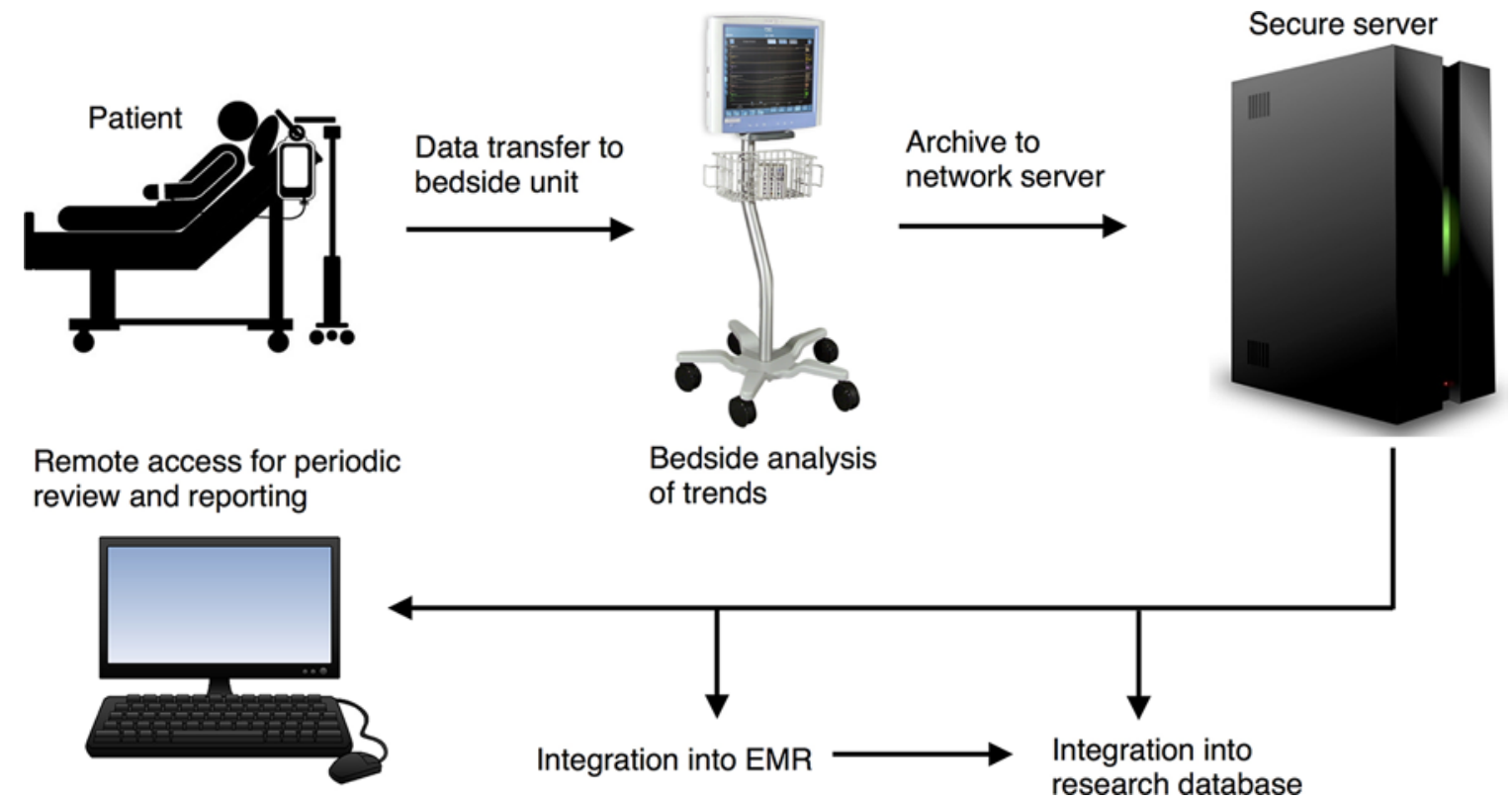

FIG. 3. Workflow diagram for recording and analysis of intracranial multimodality monitoring data. Data from various physiological monitors are recorded onto a bedside unit (e.g., Moberg CNS monitor), which enables time synchronization of data streams and bedside visualization of trends. Recorded data are then periodically archived to a secure cloud-based server for future online data review. Archived data can be directly pulled into electronic medical record (EMR) flow sheets, as well as quality, outcome, and research databases. During offline analysis, further quantitative analyses can be performed to identify both trends and physiological states that cannot be readily identified at the bedside.

and suggests that actively reducing ICP can improve brain oxygenation (Fig. 4). In contrast, ICP elevations caused by increases in cerebral perfusion would be expected to increase $\mathrm{PbtO}_{2}$, producing the opposite relationship.

Calculating the correlation between BP and CBF (or a proxy measurement) can provide a measure of cerebral autoregulation - the ability of the brain to maintain constant $\mathrm{CBF}$ in the face of changing CPP. The pressure reactivity index $(\mathrm{PRx}),{ }^{14}$ which measures time-varying changes in the correlation coefficient between arterial BP and ICP, has emerged as a widely adopted index of autoregulation. Negative correlations between ICP and BP (i.e., negative PRx) suggest intact autoregulation (because elevated BP should trigger vasoconstriction and decreased cerebral blood volume), whereas ICP values that correlate positively with BP (i.e., positive PRx) indicate failed autoregulation. Persistently positive PRx is associated with poor outcome, ${ }^{14,75}$ and maintaining $\mathrm{CPP}$ in a range where PRx is minimized

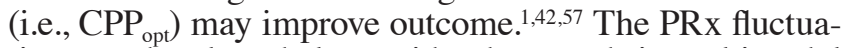
tions can be plotted along with other trends in multimodal data displays (Fig. 2), allowing the effects of different therapies on the autoregulatory state to be assessed. Similar autoregulation indices between CPP and $\mathrm{PbtO}_{2}{ }^{27}$ and CPP and $\mathrm{CBF}^{58}$ have been constructed, although they have not been validated to the same degree as PRx. ${ }^{74}$

\section{Multidimensional Approaches}

\section{Cluster Analysis}

Rather than vary smoothly over a broad range of values, variables such as ICP and $\mathrm{PbtO}_{2}$ may aggregate into distinct groups that signify specific physiological states. Cluster analysis methods can help identify these related groups in an unbiased fashion by calculating a distance metric between data points and then identifying collections of data points that lie close to each other. ${ }^{39}$ Although conceptually simple (Fig. 4), the power of cluster analysis is that it can easily be scaled to analyze relationships between large numbers of variables. In previous studies in which this approach was used to analyze physiological data from patients with multisystem trauma and TBI, novel combinations of physiological variables were found that predict infection, death, and poor outcome. ${ }^{13,55}$

\section{Multivariate Regression Models}

Predictive models based on least-squares polynomial data-fitting techniques are simple, efficient, and effective means of developing automated approaches to identify unique physiological signatures that portend poor outcome. Logistic regression modeling is one such multivariate analysis technique that can provide robust detection of worsening trends of intracranial monitoring values. ${ }^{31,33} \mathrm{Bi}-$ nomial distributions of independent data variables are created by aggregating large data sets from several patients who have known outcomes. Sufficiently large retrospective data sets will create accurate models that can be used to predict future states of new patients based on aggregates of their monitoring data plotted against the model.

\section{Smart Alarms}

Finally, there has been significant interest in the critical care field in developing alarms that provide early alerts about worsening patient conditions. ${ }^{29,53}$ Standard alarms have typically relied on simple and separate thresholds for independent data variables such as heart rate, BP, and ICP. 


\section{Correlation analysis}

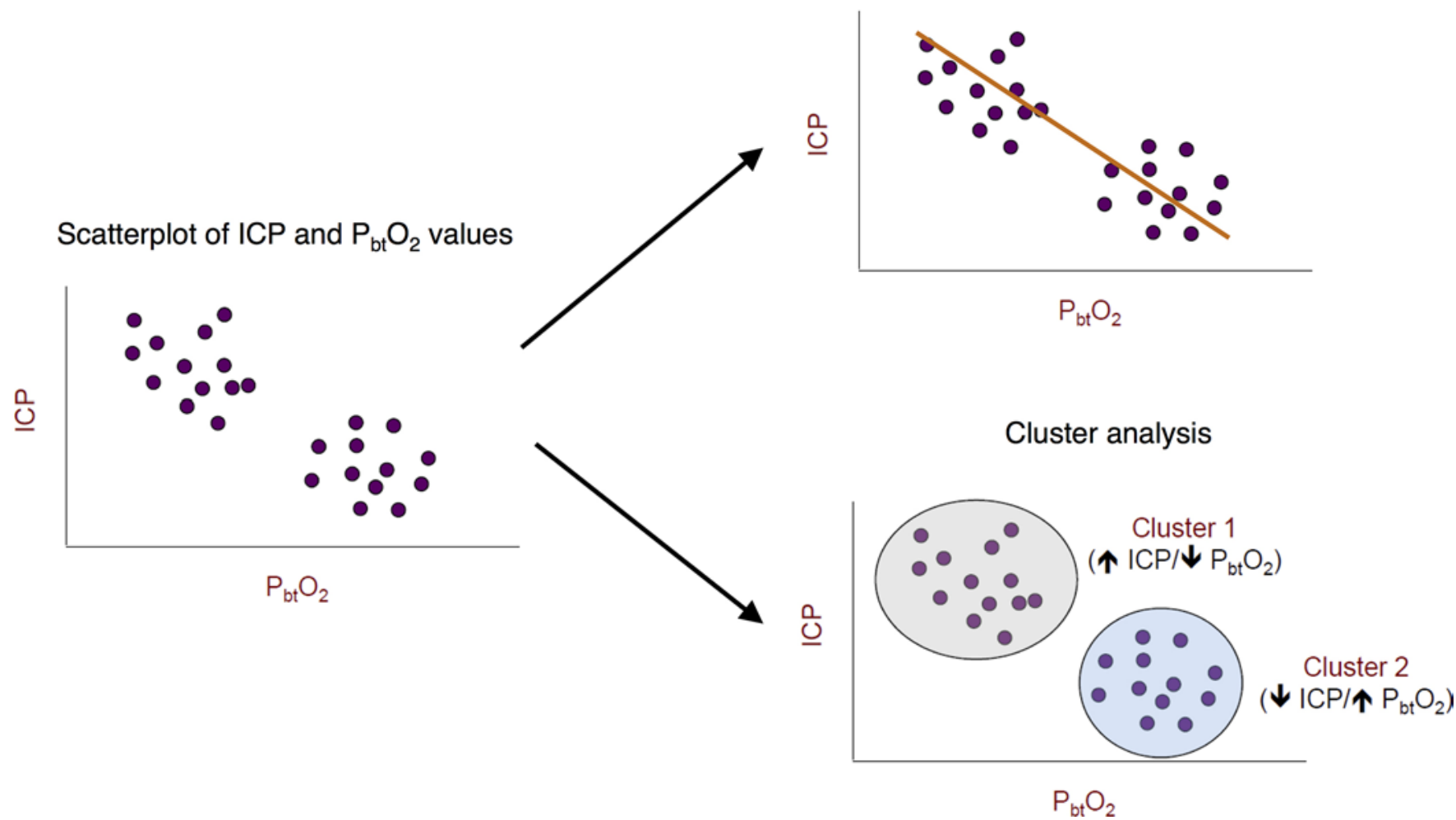

FIG. 4. Schematic of methods used to extract relationships between recorded variables. Paired ICP and $\mathrm{PbtO}_{2}$ values recorded over time and plotted are depicted in a scatterplot (left). In this hypothetical example, a relationship between ICP and $\mathrm{PbtO}_{2}$ is easily discerned, wherein higher ICP is associated with lower $\mathrm{PbtO}_{2}$. The strength of the correlation between the 2 variables (upper right) can be tracked over time and may reflect an underlying physiological process or treatment effect. The data also appear to be grouped into 2 clusters, one with high ICP and low $\mathrm{PbtO}_{2}$, and another with low ICP and higher $\mathrm{PbtO}_{2}$. Cluster analysis methods (lower right) calculate the distance between data points to identify groups that may correspond to distinct physiological states. Although depicted for only 2 variables, cluster analysis can be performed on any number of simultaneously recorded variables.

However, the large variance of clinical data leads to a large number of false-positive alarms that are not clinically relevant. Logistic regression models enable the use of more sophisticated trigger thresholds to alert clinicians toward worsening trends in monitoring data, and consequently the development of "smart alarms" that could provide sensitive and specific detection of early changes in the monitoring data while minimizing false positives.

\section{Conclusions}

Invasive multimodality monitoring holds great potential for identifying unique physiological signatures of secondary brain injury so that patient care is individualized. The increasing adoption of invasive multimodality monitoring bundles in neurocritical care units promises to usher in a new era of precision medicine. To realize this goal, however, standardized methods for data visualization and interpretation are required. In addition, the development of novel analytical methods and informatics tools that allow for unbiased assessments of large, multidimensional data sets will be crucial for the successful translation of multimodality monitoring from the realm of clinical research to standard clinical practice.

\section{References}

1. Aries MJ, Czosnyka M, Budohoski KP, Steiner LA, Lavinio A, Kolias AG, et al: Continuous determination of optimal cerebral perfusion pressure in traumatic brain injury. Crit Care Med 40:2456-2463, 2012

2. Bergsneider M, Hovda DA, Shalmon E, Kelly DF, Vespa PM, Martin NA, et al: Cerebral hyperglycolysis following severe traumatic brain injury in humans: a positron emission tomography study. J Neurosurg 86:241-251, 1997

3. Bhatia R, Hashemi P, Razzaq A, Parkin MC, Hopwood SE, Boutelle MG, et al: Application of rapid-sampling, online microdialysis to the monitoring of brain metabolism during aneurysm surgery. Neurosurgery 58 (4 Suppl 2): ONS-313ONS-321, 2006

4. Bohman LE, Pisapia JM, Sanborn MR, Frangos S, Lin E, Kumar M, et al: Response of brain oxygen to therapy correlates with long-term outcome after subarachnoid hemorrhage. Neurocrit Care 19:320-328, 2013

5. Bouma GJ, Muizelaar JP, Choi SC, Newlon PG, Young HF: Cerebral circulation and metabolism after severe traumatic brain injury: the elusive role of ischemia. J Neurosurg 75:685-693, 1991

6. Carney N, Totten AM, O'Reilly C, Ullman JS, Hawryluk GW, Bell MJ, et al: Guidelines for the management of severe traumatic brain injury, fourth edition. Neurosurgery 80:6-15, 2017

7. Chamoun R, Suki D, Gopinath SP, Goodman JC, Robertson C: Role of extracellular glutamate measured by cerebral 
microdialysis in severe traumatic brain injury. $\mathbf{J}$ Neurosurg 113:564-570, 2010

8. Chan MT, Ng SC, Lam JM, Poon WS, Gin T: Re-defining the ischemic threshold for jugular venous oxygen saturation-a microdialysis study in patients with severe head injury. Acta Neurochir Suppl 95:63-66, 2005

9. Chen HI, Stiefel MF, Oddo M, Milby AH, Maloney-Wilensky E, Frangos S, et al: Detection of cerebral compromise with multimodality monitoring in patients with subarachnoid hemorrhage. Neurosurgery 69:53-63, 2011

10. Claassen J, Jetté N, Chum F, Green R, Schmidt M, Choi H, et al: Electrographic seizures and periodic discharges after intracerebral hemorrhage. Neurology 69:1356-1365, 2007

11. Claassen J, Perotte A, Albers D, Kleinberg S, Schmidt JM, Tu B, et al: Nonconvulsive seizures after subarachnoid hemorrhage: Multimodal detection and outcomes. Ann Neurol 74:53-64, 2013

12. Clark LC Jr, Wolf R, Granger D, Taylor Z: Continuous recording of blood oxygen tensions by polarography. J Appl Physiol 6:189-193, 1953

13. Cohen MJ, Grossman AD, Morabito D, Knudson MM, Butte AJ, Manley GT: Identification of complex metabolic states in critically injured patients using bioinformatic cluster analysis. Crit Care 14:R10, 2010

14. Czosnyka M, Smielewski P, Kirkpatrick P, Laing RJ, Menon D, Pickard JD: Continuous assessment of the cerebral vasomotor reactivity in head injury. Neurosurgery 41:11-19, 1997

15. Dreier JP: The role of spreading depression, spreading depolarization and spreading ischemia in neurological disease. Nat Med 17:439-447, 2011

16. Glenn TC, Kelly DF, Boscardin WJ, McArthur DL, Vespa P, Oertel M, et al: Energy dysfunction as a predictor of outcome after moderate or severe head injury: indices of oxygen, glucose, and lactate metabolism. J Cereb Blood Flow Metab 23:1239-1250, 2003

17. Graham DI, Adams JH: Ischaemic brain damage in fatal head injuries. Lancet 1:265-266, 1971

18. Graham DI, Adams JH, Doyle D: Ischaemic brain damage in fatal non-missile head injuries. J Neurol Sci 39:213-234, 1978

19. Guillaume J, Janny P: [Continuous intracranial manometry; physiopathologic and clinical significance of the method.] Presse Med 59:953-955, 1951 (Fr)

20. Hartings JA, Bullock MR, Okonkwo DO, Murray LS, Murray GD, Fabricius M, et al: Spreading depolarisations and outcome after traumatic brain injury: a prospective observational study. Lancet Neurol 10:1058-1064, 2011

21. Hartings JA, Shuttleworth CW, Kirov SA, Ayata C, Hinzman JM, Foreman B, et al: The continuum of spreading depolarizations in acute cortical lesion development: Examining Leão's legacy. J Cereb Blood Flow Metab 37:1571-1594, 2017

22. Hemphill JC, Andrews P, De Georgia M: Multimodal monitoring and neurocritical care bioinformatics. Nat Rev Neurol 7:451-460, 2011

23. Hertle DN, Dreier JP, Woitzik J, Hartings JA, Bullock R, Okonkwo DO, et al: Effect of analgesics and sedatives on the occurrence of spreading depolarizations accompanying acute brain injury. Brain 135:2390-2398, 2012

24. Hillered L, Vespa PM, Hovda DA: Translational neurochemical research in acute human brain injury: the current status and potential future for cerebral microdialysis. J Neurotrauma 22:3-41, 2005

25. Hocker S, Nagarajan E, Rabinstein AA, Hanson D, Britton JW: Progressive brain atrophy in super-refractory status epilepticus. JAMA Neurol 73:1201-1207, 2016

26. Hutchinson PJ, Jalloh I, Helmy A, Carpenter KL, Rostami E, Bellander BM, et al: Consensus statement from the 2014 International Microdialysis Forum. Intensive Care Med 41:1517-1528, 2015

27. Jaeger M, Schuhmann MU, Soehle M, Nagel C, Meixens- berger J: Continuous monitoring of cerebrovascular autoregulation after subarachnoid hemorrhage by brain tissue oxygen pressure reactivity and its relation to delayed cerebral infarction. Stroke 38:981-986, 2007

28. Kety SS, Shenkin HA, Schmidt CF: The effects of increased intracranial pressure on cerebral circulatory functions in man. J Clin Invest 27:493-499, 1948

29. King A, Fortino K, Stevens N, Shah S, Fortino-Mullen M, Lee I: Evaluation of a smart alarm for intensive care using clinical data. Conf Proc IEEE Eng Med Biol Soc 2012:166-169, 2012

30. Ko SB, Choi HA, Parikh G, Schmidt JM, Lee K, Badjatia N, et al: Real time estimation of brain water content in comatose patients. Ann Neurol 72:344-350, 2012

31. Lang EW, Pitts LH, Damron SL, Rutledge R: Outcome after severe head injury: an analysis of prediction based upon comparison of neural network versus logistic regression analysis. Neurol Res 19:274-280, 1997

32. Lauritzen M, Dreier JP, Fabricius M, Hartings JA, Graf R, Strong AJ: Clinical relevance of cortical spreading depression in neurological disorders: migraine, malignant stroke, subarachnoid and intracranial hemorrhage, and traumatic brain injury. J Cereb Blood Flow Metab 31:17-35, 2011

33. Li YC, Liu L, Chiu WT, Jian WS: Neural network modeling for surgical decisions on traumatic brain injury patients. Int $\mathbf{J}$ Med Inform 57:1-9, 2000

34. Lundberg N, Troupp H, Lorin H: Continuous recording of the ventricular-fluid pressure in patients with severe acute traumatic brain injury. A preliminary report. J Neurosurg 22:581-590, 1965

35. Maas AI, Stocchetti N, Bullock R: Moderate and severe traumatic brain injury in adults. Lancet Neurol 7:728-741, 2008

36. Macdonald RL: Delayed neurological deterioration after subarachnoid haemorrhage. Nat Rev Neurol 10:44-58, 2014

37. Macmillan CS, Andrews PJ: Cerebrovenous oxygen saturation monitoring: practical considerations and clinical relevance. Intensive Care Med 26:1028-1036, 2000

38. Maloney-Wilensky E, Gracias V, Itkin A, Hoffman K, Bloom $\mathrm{S}$, Yang W, et al: Brain tissue oxygen and outcome after severe traumatic brain injury: a systematic review. Crit Care Med 37:2057-2063, 2009

39. McLachlan GJ: Cluster analysis and related techniques in medical research. Stat Methods Med Res 1:27-48, 1992

40. Miller JD, Becker DP, Ward JD, Sullivan HG, Adams WE, Rosner MJ: Significance of intracranial hypertension in severe head injury. J Neurosurg 47:503-516, 1977

41. Nangunoori R, Maloney-Wilensky E, Stiefel M, Park S, Andrew Kofke W, Levine JM, et al: Brain tissue oxygen-based therapy and outcome after severe traumatic brain injury: a systematic literature review. Neurocrit Care 17:131-138, 2012

42. Needham E, McFadyen C, Newcombe V, Synnot AJ, Czosnyka M, Menon D: Cerebral perfusion pressure targets individualized to pressure-reactivity index in moderate to severe traumatic brain injury: a systematic review. J Neurotrauma 34:963-970, 2017

43. Oddo M, Levine JM, Frangos S, Maloney-Wilensky E, Carrera E, Daniel RT, et al: Brain lactate metabolism in humans with subarachnoid hemorrhage. Stroke 43:1418-1421, 2012

44. Oddo M, Levine JM, Mackenzie L, Frangos S, Feihl F, Kasner SE, et al: Brain hypoxia is associated with short-term outcome after severe traumatic brain injury independently of intracranial hypertension and low cerebral perfusion pressure. Neurosurgery 69:1037-1045, 2011

45. Ramakrishna R, Stiefel M, Udoetuk J, Spiotta A, Levine JM, Kofke WA, et al: Brain oxygen tension and outcome in patients with aneurysmal subarachnoid hemorrhage. J Neurosurg 109:1075-1082, 2008 (Erratum in J Neurosurg 110:613, 2009)

46. Robertson CS, Gopinath SP, Goodman JC, Contant CF, Va- 
ladka $\mathrm{AB}$, Narayan $\mathrm{RK}: \mathrm{SjvO}_{2}$ monitoring in head-injured patients. J Neurotrauma 12:891-896, 1995

47. Rogers ML, Leong CL, Gowers SA, Samper IC, Jewell SL, Khan A, et al: Simultaneous monitoring of potassium, glucose and lactate during spreading depolarization in the injured human brain - Proof of principle of a novel real-time neurochemical analysis system, continuous online microdialysis. J Cereb Blood Flow Metab 37:1883-1895, 2017

48. Rosenfeld JV, Maas AI, Bragge P, Morganti-Kossmann MC, Manley GT, Gruen RL: Early management of severe traumatic brain injury. Lancet 380:1088-1098, 2012

49. Rosenthal G, Hemphill JC III, Sorani M, Martin C, Morabito $\mathrm{D}$, Obrist WD, et al: Brain tissue oxygen tension is more indicative of oxygen diffusion than oxygen delivery and metabolism in patients with traumatic brain injury. Crit Care Med 36:1917-1924, 2008

50. Rosenthal G, Sanchez-Mejia RO, Phan N, Hemphill JC III, Martin C, Manley GT: Incorporating a parenchymal thermal diffusion cerebral blood flow probe in bedside assessment of cerebral autoregulation and vasoreactivity in patients with severe traumatic brain injury. J Neurosurg 114:62-70, 2011

51. Schell RM, Cole DJ: Cerebral monitoring: jugular venous oximetry. Anesth Analg 90:559-566, 2000

52. Schmidt JM, Ko SB, Helbok R, Kurtz P, Stuart RM, Presciutti $\mathrm{M}$, et al: Cerebral perfusion pressure thresholds for brain tissue hypoxia and metabolic crisis after poor-grade subarachnoid hemorrhage. Stroke 42:1351-1356, 2011

53. Siebig S, Kuhls S, Imhoff M, Langgartner J, Reng M, Schölmerich J, et al: Collection of annotated data in a clinical validation study for alarm algorithms in intensive care-a methodologic framework. J Crit Care 25:128-135, 2010

54. Skjøth-Rasmussen J, Schulz M, Kristensen SR, Bjerre P: Delayed neurological deficits detected by an ischemic pattern in the extracellular cerebral metabolites in patients with aneurysmal subarachnoid hemorrhage. J Neurosurg 100:8-15, 2004

55. Sorani MD, Hemphill JC III, Morabito D, Rosenthal G, Manley GT: New approaches to physiological informatics in neurocritical care. Neurocrit Care 7:45-52, 2007

56. Stein NR, McArthur DL, Etchepare M, Vespa PM: Early cerebral metabolic crisis after TBI influences outcome despite adequate hemodynamic resuscitation. Neurocrit Care 17:49-57, 2012

57. Steiner LA, Czosnyka M, Piechnik SK, Smielewski P, Chatfield D, Menon DK, et al: Continuous monitoring of cerebrovascular pressure reactivity allows determination of optimal cerebral perfusion pressure in patients with traumatic brain injury. Crit Care Med 30:733-738, 2002

58. Tackla R, Hinzman JM, Foreman B, Magner M, Andaluz N, Hartings JA: Assessment of cerebrovascular autoregulation using regional cerebral blood flow in surgically managed brain trauma patients. Neurocrit Care 23:339-346, 2015

59. Takasu A, Yagi K, Ishihara S, Okada Y: Combined continuous monitoring of systemic and cerebral oxygen metabolism after cardiac arrest. Resuscitation 29:189-194, 1995

60. Timofeev I, Carpenter KL, Nortje J, Al-Rawi PG, O’Connell MT, Czosnyka M, et al: Cerebral extracellular chemistry and outcome following traumatic brain injury: a microdialysis study of 223 patients. Brain 134:484-494, 2011

61. Tisdall MM, Smith M: Cerebral microdialysis: research technique or clinical tool. Br J Anaesth 97:18-25, 2006

62. Vajkoczy P, Horn P, Thome C, Munch E, Schmiedek P: Regional cerebral blood flow monitoring in the diagnosis of delayed ischemia following aneurysmal subarachnoid hemorrhage. J Neurosurg 98:1227-1234, 2003

63. Vajkoczy P, Roth H, Horn P, Lucke T, Thomé C, Hubner U, et al: Continuous monitoring of regional cerebral blood flow: experimental and clinical validation of a novel thermal diffusion microprobe. J Neurosurg 93:265-274, 2000

64. Vespa P, Bergsneider M, Hattori N, Wu HM, Huang SC, Mar- tin NA, et al: Metabolic crisis without brain ischemia is common after traumatic brain injury: a combined microdialysis and positron emission tomography study. J Cereb Blood Flow Metab 25:763-774, 2005

65. Vespa P, Prins M, Ronne-Engstrom E, Caron M, Shalmon E, Hovda DA, et al: Increase in extracellular glutamate caused by reduced cerebral perfusion pressure and seizures after human traumatic brain injury: a microdialysis study. J Neurosurg 89:971-982, 1998

66. Vespa P, Tubi M, Claassen J, Buitrago-Blanco M, McArthur $D$, Velazquez AG, et al: Metabolic crisis occurs with seizures and periodic discharges after brain trauma. Ann Neurol 79:579-590, 2016

67. Vespa PM, McArthur DL, Xu Y, Eliseo M, Etchepare M, Dinov I, et al: Nonconvulsive seizures after traumatic brain injury are associated with hippocampal atrophy. Neurology 75:792-798, 2010

68. Vespa PM, Miller C, McArthur D, Eliseo M, Etchepare M, Hirt D, et al: Nonconvulsive electrographic seizures after traumatic brain injury result in a delayed, prolonged increase in intracranial pressure and metabolic crisis. Crit Care Med 35:2830-2836, 2007

69. Vespa PM, Nuwer MR, Nenov V, Ronne-Engstrom E, Hovda DA, Bergsneider M, et al: Increased incidence and impact of nonconvulsive and convulsive seizures after traumatic brain injury as detected by continuous electroencephalographic monitoring. J Neurosurg 91:750-760, 1999

70. Vespa PM, O'Phelan K, McArthur D, Miller C, Eliseo M, Hirt D, et al: Pericontusional brain tissue exhibits persistent elevation of lactate/pyruvate ratio independent of cerebral perfusion pressure. Crit Care Med 35:1153-1160, 2007

71. Waziri A, Claassen J, Stuart RM, Arif H, Schmidt JM, Mayer SA, et al: Intracortical electroencephalography in acute brain injury. Ann Neurol 66:366-377, 2009

72. Witsch J, Frey HP, Schmidt JM, Velazquez A, Falo CM, Reznik M, et al: Electroencephalographic periodic discharges and frequency-dependent brain tissue hypoxia in acute brain injury. JAMA Neurol 74:301-309, 2017

73. Wolf S, Vajkoczy P, Dengler J, Schürer L, Horn P: Drift of the Bowman Hemedex ${ }^{\circledR}$ cerebral blood flow monitor between calibration cycles. Acta Neurochir Suppl 114:187-190, 2012

74. Zeiler FA, Donnelly J, Menon DK, Smielewski P, Zweifel C, Brady K, et al: Continuous autoregulatory indices derived from multi-modal monitoring: each one is not like the Other. J Neurotrauma [epub ahead of print], 2017

75. Zweifel C, Lavinio A, Steiner LA, Radolovich D, Smielewski P, Timofeev I, et al: Continuous monitoring of cerebrovascular pressure reactivity in patients with head injury. Neurosurg Focus 25(4):E2, 2008

\section{Disclosures}

The authors report no conflict of interest concerning the materials or methods used in this study or the findings specified in this paper.

\section{Author Contributions}

Conception and design: Balu, Sinha. Analysis and interpretation of data: Balu, Sinha, Hudgins. Drafting the article: Balu, Sinha, Hudgins. Critically revising the article: all authors. Reviewed submitted version of manuscript: all authors. Approved the final version of the manuscript on behalf of all authors: Balu.

\section{Correspondence}

Ramani Balu, Department of Neurology, Division of Neurocritical Care, Perelman School of Medicine, University of Pennsylvania, 3W Gates, 3600 Spruce St., Philadelphia, PA 19104. email: ramani.balu@uphs.upenn.edu. 